\title{
Postpartum Placental Separation
}

National Cancer Institute

\section{Source}

National Cancer Institute. Postpartum Placental Separation. NCI Thesaurus. Code C92854.

A process in childbirth in which the placenta separates from its uterine lining, after the delivery of the baby. 